\title{
Noise Performance of Time Stretch System with Distributed and Discrete Amplifiers
}

\author{
Salih K. Kalyoncu, Yuewang Huang, En-Kuang Tien, Enver Adas, Dogukan Yildirim and Ozdal Boyraz \\ EECS Department, University of California, Irvine, 92697, USA
}

Email: oboyraz@uci.edu

\begin{abstract}
We analyzed the noise performance of time stretched analog to digital converter with both lumped and distributed amplification cases. We show that distributed amplification provides up to $16 \mathrm{~dB}$ higher SNR corresponding to $\sim 2.5$ bit higher resolution.

OCIS codes: (250.4745) Optical processing devices; (320.7085) Ultrafast information processing; (070.4340) Nonlinear optical signal processing;
\end{abstract}

\section{Introduction}

The revolution of digital signal processing has enabled many applications ranging from communications to radar systems by introducing high speed operations. The demand for ultrafast signal processing requires capturing of broadband signals [1,2]. Photonic analog to digital converters (ADCs) which aim to improve the limiting properties of electronic ADCs, have been investigated to increase the analog bandwidth and sampling rates and to facilitate capturing of broadband analog signals [3]. In particular, recent advances in optical signal processing have enabled the design of ultrafast ADCs with high bandwidth and sampling rates up to $10 \mathrm{THz}$ by using time stretch technique [4]. However, the cumulative noise mainly due to amplifier, double Rayleigh scattering (DRS) and relative intensity noise (RIN) from the pumps limit the maximum effective number of bits (ENOB) that can be achieved in such a system. In this paper we analyze the noise performance and hence the ENOB performance of time stretch ADCs with distributed and lumped amplifications. We show that time stretch system with $>10 \mathrm{GHz}$ analog bandwidth exhibit 16dB higher SNR corresponding to $\sim 2.5$ bit higher resolution.

\section{Noise Analysis and ENOB calculation}

Time stretched ADC with stretch factor of 40 is designed to capture $10 \mathrm{GHz}$ analog signals by $12 \mathrm{bit} 250 \mathrm{MHz}$ high fidelity electronic digitizers. Time stretching is achieved by propagating a broadband continuum signal with $60 \mathrm{~nm}$ bandwidth centered at $1590 \mathrm{~nm}$ through the dispersion compensation modules (DCMs). Time stretch system consists of two DCMs where the first DCM has dispersion value of $-32 \mathrm{ps} / \mathrm{nm}$ and the second module has the total dispersion value of $-1258 \mathrm{ps} / \mathrm{nm}$. In particular, the second module consists of three dispersion compensation fiber modules, Fig. 1. The system performance of the ADC in terms of ENOB is limited by the noise cumulated in the system from both optical and electrical sources. A deep analysis is conducted to calculate the signal to noise and distortion ratio (SINAD) and ENOB for both discrete and distributed amplification configurations. Under SINAD analysis, all the noise components such as amplified spontaneous emission (ASE), relative intensity noise (RIN), double Rayleigh backscattering (DRS), quantization noise and the detector noises as thermal and shot noises are investigated individually. For the distributed amplification, a flat gain Raman amplifier $(<0.5 \mathrm{~dB}$ gain ripple) over $60 \mathrm{~nm}$ wide is designed to provide a uniform amplification for the broadband signal, Fig. 1b. Additionally, two different configurations for lumped amplification including single EDFA case and double EDFA cases are investigated.
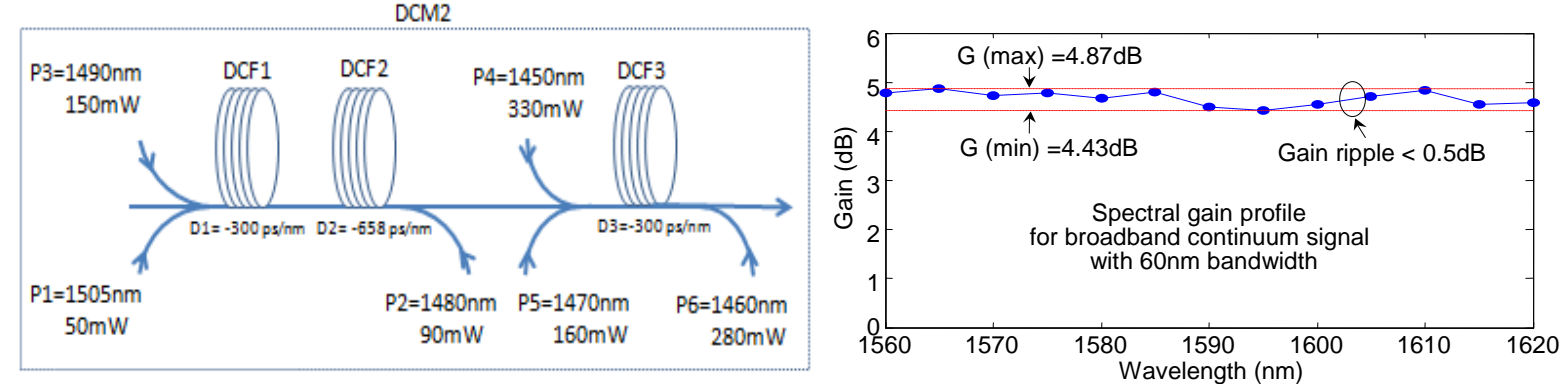

Fig. 1. The spectra of pump configuration for the Raman amplifier with required wavelengths and the powers (1a) to obtain the flat wideband gain profile shown on the right (1b).

RIN transfer in distributed amplifiers has been identified as one of the main contributor for low SINAD time stretch system. Here we analyze the RIN transfer of the designed Raman amplifiers and show that RIN transfer from pump to signal varies between $6 \mathrm{~dB}$ and $11 \mathrm{~dB}$ over the entire $60 \mathrm{~nm}$ bandwidth, Fig. $2 \mathrm{a}$. In addition to RIN transfer, we individually include the contributions from double Rayleigh backscattering, shot noise, thermal noise, quantization noise and ASE noise. As indicated in Figure 2b, most noise accumulation occurs at low frequencies that 


\section{CTuA7.pdf}

can be eliminated by using high pass filter such as $10 \mathrm{MHz}$ and $100 \mathrm{MHz}$ used in these simulations that limits the lower edge of the analog bandwidth of the time stretch system to $400 \mathrm{MHz}$ and $4 \mathrm{GHz}$, respectively.
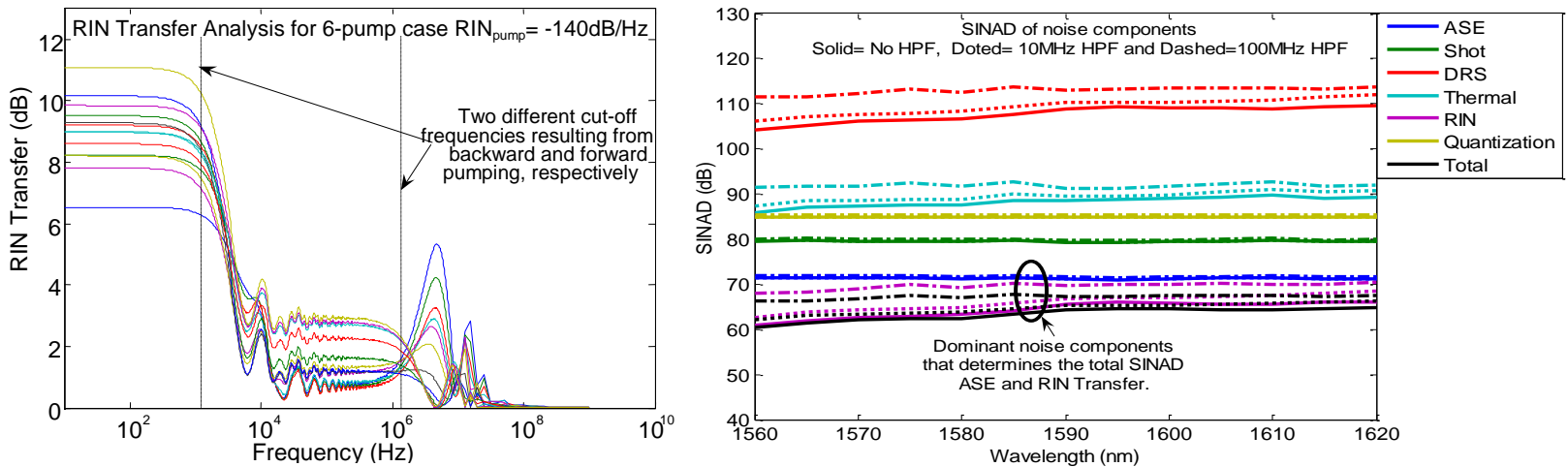

Fig. 2. The RIN transfer (2a) from the pumps to the WDM signals in 6 pumped Raman amplifier shown in Fig. 1a and the individual noise components effective in the distributed amplification (2b).

Similarly, the same noise analysis is performed for time stretch system with discrete amplifiers. To have fair comparison, we insert discrete amplifiers that produce the same signal power at the detector as the Raman amplifier and assume $5 \mathrm{~dB}$ noise figure for each amplifier. In the first case we assume a single EDFA with $11.5 \mathrm{~dB}$ gain inserted after DCF3. In the second case we insert double amplifiers after DCF2 and DCF3. Effective noise figure of the distributed and discrete amplifiers are illustrated in Fig. 3a. Figure 3b illustrates the SINAD values and corresponding ENOB values for distributed and discrete amplifiers. SINAD values for distributed amplification varies from $60 \mathrm{~dB}$ to $68 \mathrm{~dB}$. The biggest noise component is generated by RIN transfer from pump lasers in distributed amplification. Additionally, double Rayleigh scattering is determined to be detrimental for high ENOB systems. In discrete amplification case we obtain SINAD values of $\sim 55 \mathrm{~dB}$. The biggest noise component in discrete amplification is produced by ASE noise. In particular, we obtain $\sim 16 \mathrm{~dB}$ higher SINAD for distributed amplification that can result in $\sim 2.5$ bit higher resolution, Fig. $3 b$.
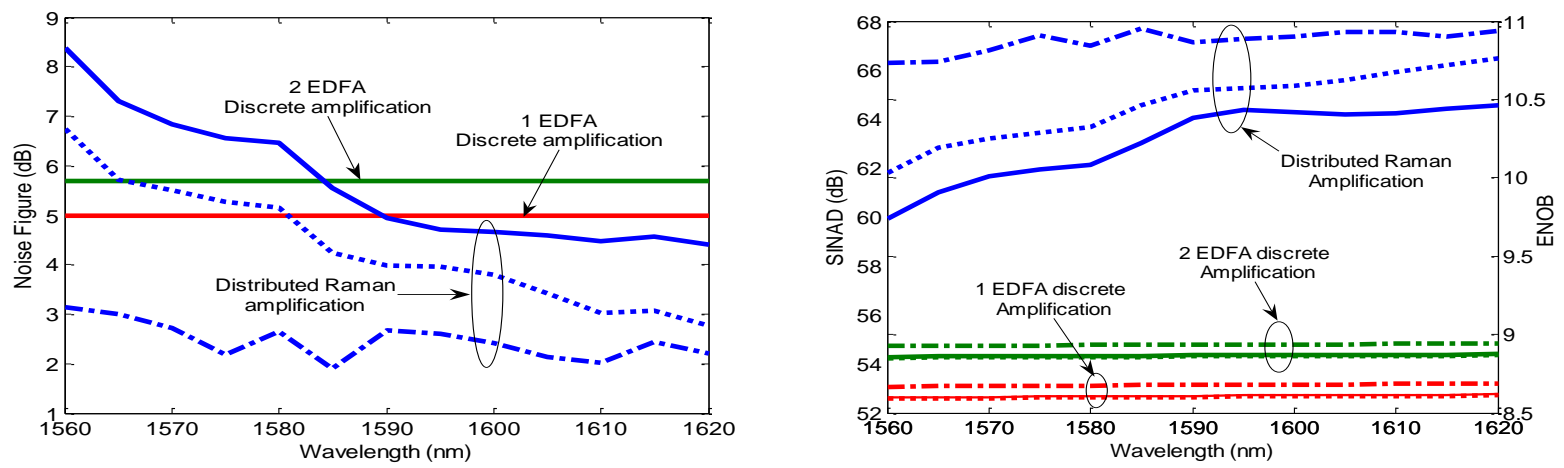

Fig. 3. The Noise figure (3a) and the ENOB (3b) calculation of both discrete and distributed amplification schemas for ADC with the sampling rate $\mathrm{f}_{\mathrm{s}}=3 \mathrm{GHz}$, bandwidth $\mathrm{BW}=1 \mathrm{GHz}$ and the quantization bits $\mathrm{N}=12 \mathrm{bit}$. Solid= No HPF, Doted $=10 \mathrm{MHz} \mathrm{HPF}$, Dashed=100MHz HPF.

\section{Conclusion}

We analyze the noise performance and hence the ENOB performance of time stretch ADCs with distributed and lumped amplifications. We conclude that time stretch system with distributed amplification is limited by RIN transfer and discrete amplification system is limited by ASE noise. Overall, we estimate that distributed amplification in time stretch system with $>10 \mathrm{GHz}$ analog bandwidth exhibit up to $16 \mathrm{~dB}$ higher SNR corresponding to $\sim 2.5$ bit higher resolution.

\section{Acknowledgement}

This work is supported by DARPA RADER program (grant \# HR0011-10-C-0063).

\section{References}

[1] J. A. Wepman, “Analog-to-digital converters and their applications in radio receivers," IEEE Commun. Mag., Vol. 33, pp. 39-45, May 1995. [2] Y. Han and B. Jalali, "Photonic Time-Stretched Analog-to-Digital Converter: Fundamental Concepts and Practical Considerations", Journal of Lightwave Tech., Vol. 21, No. 12, Dec 2003.

[3] G. C. Valley, "Photonic analog to digital converters", Optics Express Vol. 15, No. 5, March 2007.

[4] J. Chou, O. Boyraz, B. Jalali, “Femtosecond real-time single-shot digitizer,” Applied Physics Letters 91, 161105_2007. 